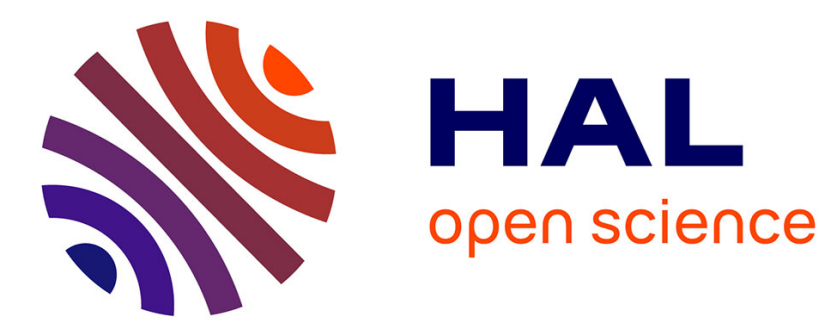

\title{
Qualifier pénalement l'inceste: les incertitudes du droit pénal français contemporain
}

\author{
Marie Romero
}

\section{To cite this version:}

Marie Romero. Qualifier pénalement l'inceste: les incertitudes du droit pénal français contemporain. Cahiers d'anthropologie sociale, 2017, La peur de l'inceste, 15, pp.127-143. hal-01749605

\section{HAL Id: hal-01749605 https://hal-amu.archives-ouvertes.fr/hal-01749605}

Submitted on 29 Mar 2018

HAL is a multi-disciplinary open access archive for the deposit and dissemination of scientific research documents, whether they are published or not. The documents may come from teaching and research institutions in France or abroad, or from public or private research centers.
L'archive ouverte pluridisciplinaire HAL, est destinée au dépôt et à la diffusion de documents scientifiques de niveau recherche, publiés ou non, émanant des établissements d'enseignement et de recherche français ou étrangers, des laboratoires publics ou privés. 


\title{
Table des matières
}

Introduction

1. L'inceste dans le droit pénal français : complexité de la règle ....................................................2

1.1 Les premiers codes pénaux français : le silence du droit face à l'inceste ................................... 2

1.2 Code pénal contemporain : la nouvelle référence au consentement ........................................2

2. Qualifier pénalement l'inceste : incertitudes dans l'application du droit ..................................... 3

2.1 Les dispositions du droit pénal pour viser les liens de famille incestueux ................................ 4

2.1.1 Usage du sur-qualificatif incestueux : une conception minimaliste de l'inceste .................................... 4

2.1.2 Critère aggravant d'ascendant ou de personne ayant autorité : un usage disparate............................... 4

2.2 Prouver le non-consentement du mineur victime d'inceste : prévalence de l'âge.......................5

2.2.1 Le jeune âge de l'enfant : un seuil irréfragable de non-consentement ................................................ 6

2.2.2 Autres âges de la minorité : incertitudes des seuils ...................................................................... 7

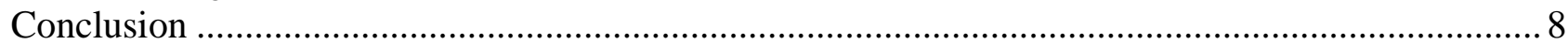

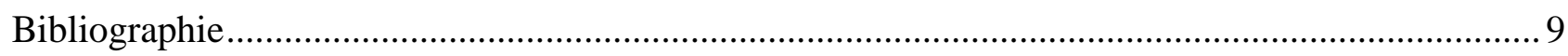

\section{Qualifier pénalement l'inceste : les incertitudes du droit pénal français contemporain}

\author{
Marie Romero
}

\section{Introduction}

L'inceste désigne des «relations sexuelles entre des personnes qui sont parentes ou alliées au degré prohibé par les lois et la coutume ${ }^{\mathrm{i}} »$. Le droit a suivi ce postulat anthropologique limitant les unions matrimoniales et sexuelles (Godelier, 2004 : 617) mais sa répression a varié selon les époques et les systèmes de parenté. Autrefois crime spécifique puni de mort, l'inceste n'est plus sanctionné en tant que tel dans le droit pénal français contemporain. Il n'est qu'une circonstance aggravante dans le viol et de l'atteinte sexuelle avec ou sans violence, tenant à la qualité d'ascendant ou de personne ayant autorité sur la victime ${ }^{\mathrm{ii}}$.

Cependant, en faisant de l'inceste un cas particulier des infractions sexuelles, le viol est devenu sa forme la plus grave $e^{\mathrm{iii}}$, générant le sentiment d'horreur le plus répulsif. En outre, en visant les liens de famille à partir du critère d'ascendant ou de personne ayant autorité sur le mineur, l'inceste se pense désormais en droit pénal dans un modèle généalogique, de filiation ou assimilé. Or, qu'en estil des liens de collatéralité (frère, cousin) ou ceux issus des familles recomposées ? N'assiste-t-on pas à une érosion du champ de la prohibition face aux bouleversements de la structure familiale (Porqueres, 2013) ? Le tabou de l'inceste est-t-il en perte de puissance (Ambroise-Rendu, 2012) ou objet de métamorphoses sociétales?

Cet article a pour but de comprendre les transformations qui ont conduit au resserrement de l'interdit de l'inceste dans le code pénal. Je propose d'en analyser les contours, mais aussi les changements à l'œuvre, en mesurant les écarts entre le droit et son application dans les tribunaux. Pour réaliser ce travail, je m'inscris dans une double lignée : l'analyse compréhensive de Marx Weber en étudiant le droit comme il agit et non comme il se pense ; la sociologie judiciaire de Jean Carbonnier, en révélant la complexité des règles et les questions non résolues à travers la mise en œuvre pratique du droit.

Mon enquête qualitative porte sur 27 affaires de violences sexuelles intrafamiliales sur mineurs, jugées en tribunal correctionnel et pour enfant au cours de l'année $2010^{\mathrm{iv}}$. Ces dossiers constituent un observatoire inédit du traitement pénal de l'inceste. Pour des raisons méthodologiques, le corpus est limité aux délits sexuels ; ne sont pas inclus les affaires en cours, en appel, classés sans suite, et les viols relevant des assises. 
La première partie de cet article présente l'analyse des transformations du droit qui ont mené au traitement pénal actuel de l'inceste, en montrant le passage d'un ordre sexuel matrimonial à un ordre post-matrimonial basé sur la nouvelle référence au consentement. La deuxième partie plonge au cœur des pratiques judiciaires confrontées aux incertitudes dans la qualification pénale de l'inceste. Je vérifierai mon hypothèse d'une recomposition fondamentale du permis et de l'interdit en matière sexuelle à partir de la barrière sacralisée des âges.

\section{L'inceste dans le droit pénal français : complexité de la règle}

\subsection{Les premiers codes pénaux français : le silence du droit face à l'inceste}

L'Ancien Régime chrétien était fondé sur un ordre naturel divin qui protégeait l'honneur des familles nobles, la sacralité du mariage et la fille impubère. L'inceste était considéré comme un péché de chair qui enveloppait les protagonistes dans la même faute au point d'abolir la référence possible au viol. Crime spécifique, il était sévèrement puni selon les degrés de parenté ou d'alliance. Ainsi, les incestes entre père et fille, entre mère et fils, entre frère et sœur, étaient passibles de la peine capitale par le feu ; les incestes entre beaux-frères et belles-sœurs, oncles et nièces, cousins, n'étaient condamnés que d'une peine arbitraire (Knibiehler, $2001: 22)$.

Après la révolution, tout change. La nouvelle approche du droit tente de libérer la loi de toute emprise religieuse (Vigarello, 1998 ; Giulianni, 2009). L'inceste entre adultes consentants relève désormais d'un interdit d'ordre moral et n'est plus sanctionné par la loi (Giuliani, 2014 ; Knibiehler, 2001 : 27). Il disparaît du premier code pénal élaboré par l'Assemblée Législative en 1791, puis réapparaît timidement dans le code pénal napoléonien de 1810 à travers la catégorie de personne ayant autorité, circonstance aggravante du viol et de l'attentat à la pudeur ${ }^{\mathrm{v}}$. S'y ajoute en 1832, celle d'ascendant, visant explicitement la parenté. Parallèlement entre le critère d'âge, à la fois comme critère aggravant de ces infractions mais aussi constitutif du nouvel attentat à la pudeur sans violence sur mineur de moins de $11 \mathrm{ans}^{\mathrm{vi}}$. C'est un bouleversement complet pour la définition de la prohibition où prévaut l'âge plus que le lien de famille ${ }^{\mathrm{vii}}$.

Contrairement à l'Ancien Régime, l'inceste ne repose plus sur l'horreur des unions consanguines mais sur les lois naturelles de la pudeur. Ce que l'on punit dans l'inceste c'est d'abord l'atteinte portée à un ordre naturel de la famille conjugale (Théry, 2010, 2016). Ces justifications nouvelles fondent les prohibitions matrimoniales du code civil (Courtois, $2004: 3$; Godelier, 2004 : 348) et bouleversent les interdits sexuels du code pénal. Ils relèvent désormais d'un modèle généalogique fondé sur une règle fondamentale : l'ordre qui régit la suite des générations et les fonctions conférées à chacune (Héritier, 1996). Aussi, assiste-t-on à un nouvel ordre juridique matrimonial bourgeois, fortement hiérarchisé, qui fait de son foyer, une sphère d'intimité où règne l'autorité du pater familias, un domaine privé où le droit pénal ne légifère pas (Poumarède, 1987). L'inceste est donc replié dans le secret des familles (Salas, 1998), rarement dénoncé en justice (Kiener, Camdessus, 1993 ; Vigarello, 1998 ; Ambroise-Rendu, 2012) et toujours entaché de soupçons sur l'enfant (Demartini, 2009).

\subsection{Code pénal contemporain : la nouvelle référence au consentement}

A la fin du $X X X^{\text {ème }}$ siècle, l'ordre juridique matrimonial fondé sur la hiérarchie des sexes et des âges implose dans les années 1960/1970 et fait voler en éclat les structures traditionnelles de la famille (Théry, 1993 ; Godelier, 2004). Parallèlement, s'engage une nouvelle approche du droit plus égalitaire, centrée sur l'individu, qui ne condamne plus la transgression d'une morale sociale mais l'atteinte au consentement de la personne (Mayer, 1981 ; Théry, 2002). La définition du viol ${ }^{\text {viii }}$, issue de la loi n80-1041 du 23 décembre 1980, marque symboliquement l'entrée nouvelle dans un ordre juridique post-matrimonial. Le nouveau code pénal de 1994 le réaffirme et fait du consentement la summa diviso des infractions sexuelles (Rassat, 2008): avec d'un côté les 
infractions qui relèvent de relations sexuelles interdites parce qu'elles violent le consentement de la personne ( viol $^{\text {ix }}$ et agression sexuelle ${ }^{\mathrm{X}}$ ) et de l'autre, celles prohibées parce que l'âge du mineur victime ne permet pas un consentement valable (atteinte sexuelle sur mineur ${ }^{\mathrm{xi}}$ ). Encore inachevées et peu comprises, ces métamorphoses soulèvent de nouveaux questionnements et suscitent des dilemmes face à l'inceste.

La loi $\mathrm{n}^{\circ} 98-468$ du 17 juin 1998 relative à la prévention et à la répression des infractions sexuelles ainsi qu'à la protection des mineurs, et la loi n²013-711 du 5 août 2013, ont allongé les délais de prescription et augmenté l'ensemble des peines, à l'exception du viol. Ainsi, l'agression sexuelle et l'atteinte sexuelle sur mineur de 15 ans et par ascendant ou personne ayant autorité, voient leurs peines passées respectivement de 7 et 5 ans, à 10 ans d'emprisonnement. En outre, la loi $\mathrm{n}^{\circ} 2010-121$ du 8 février 2010 a fait entrer le terme inceste dans le code pénal au travers du surqualificatif «incestueux» ${ }^{\text {xii }}$ ajoutée à l'infraction. Or, l'étendue de son périmètre familial a fait débat $^{\text {xiii }}$ et l'attention exclusive portée aux victimes mineures est questionnée (Terryn, 2013). Ainsi une agression sexuelle d'un frère sur sa sœur mineure âgée de 14 ans est qualifiée d'incestueuse, mais pas lorsque la même victime est âgée de 18 ans. Enfin, le législateur a tenté d'assouplir le régime de la preuve du non-consentement de la victime d'inceste en donnant une nouvelle base légale à la contrainte morale ${ }^{\mathrm{xiv}}$ mais sur fond de nombreuses controverses judiciaires. Ces nouvelles dispositions pénales mettent en évidence la façon singulière de penser l'inceste en droit pénal, qui était déjà à l'œuvre dans l'ancien droit, un rapport inégal majeur/mineur (Martial, 1996 : 93).

\section{Qualifier pénalement l'inceste : incertitudes dans l'application du droit}

Dans notre échantillon de 27 affaires, impliquant 28 auteurs présumés et 37 victimes mineures présumées, nous avons une représentation assez classique de ce que l'on trouve dans d'autres enquêtes sociologiques sur les violences sexuelles (Le Goaziou, 2011 ; Finkelhor, 1994) : une large majorité d'hommes ou de garçons mineurs auteurs $(99 \%)$ et une large proportion de filles ou fillettes victimes $(70 \%)$. Une part non négligeable concerne les garçons victimes. A partir de nos dossiers, nous avons constitué une typologie de 38 liens de famille. Ils sont présentés dans le tableau ci-dessous :

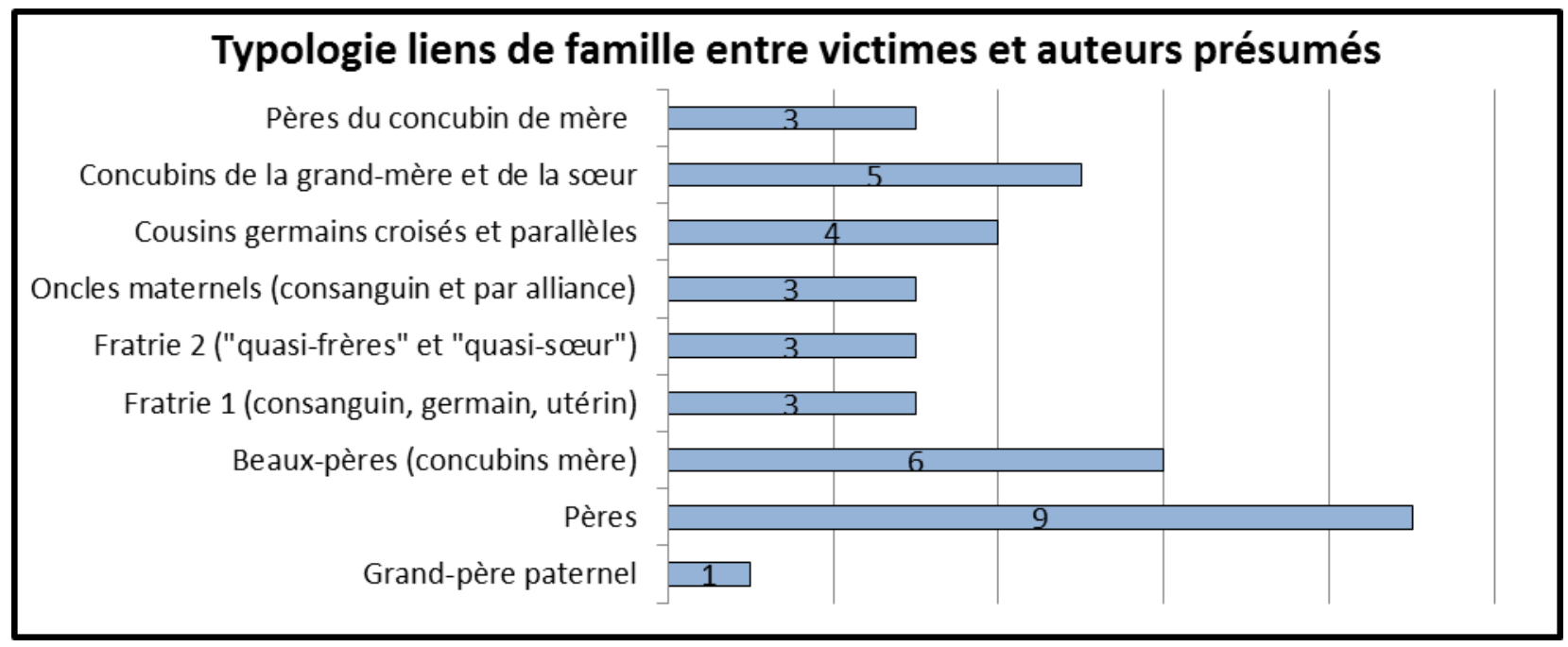

Plus de la moitié des liens incestueux sont issus de la parenté en ligne directe (pères) ou collatérale (frères, oncles ou cousins), l'autre, sont des liens péri-incestueux, non fondés sur le sang: beau-père, "quasi-frères ou sœurs " ${ }^{\mathrm{xv}}$, père du beau-père, concubin de la grand-mère, concubin de la sœur. Proportionnellement, les plus nombreux sont les liens de parenté et d'alliance en ligne directe (pères, grand-père, beaux-pères), ce qui corrobore les travaux de Giles-Sims et Finkelhor, sur les cas d'inceste (1984 : 408). 


\subsection{Les dispositions du droit pénal pour viser les liens de famille incestueux}

Nous présentons ici les deux modalités de qualification pénale de l'inceste : par l'ajout du surqualificatif «incestueux » et de l'aggravation d'ascendant ou de personne ayant autorité au sein de la même infraction.

\subsubsection{Usage du sur-qualificatif incestueux : une conception minimaliste de l'inceste}

Le droit permet de qualifier d'incestueux le viol, l'agression sexuelle ou l'atteinte sexuelle commis sur un mineur, conformément aux articles 222-31-1 et 227-27-2 du code pénal, lorsqu'ils sont commis par: l'ascendant, le frère ou la sœur, l'oncle ou la tante, le neveu ou la nièce, ou toute personne au sein de la famille qui a autorité de droit ou de fait sur le mineur. Ce vaste périmètre, dépassant largement le cadre des prohibitions matrimoniales prévues dans le code civil ${ }^{\text {xvi }}$, s'intègre à notre typologie de liens familiaux incestueux.

Or, tous les liens ne sont pas visés et certains le sont plus que d'autres. En effet, lorsqu'un père est mis en cause, le sur-qualificatif «incestueux» a été systématiquement ajouté à la qualification. A l'inverse, dès que l'on sort de cette parenté en ligne directe, il n'est plus retenu. Les professionnels n'ont pas considéré comme incestueux, les infractions sexuelles commises par un beau-père, père du beau-père, concubin de la grand-mère ou de la sœur, un frère, et enfin un cousin. Les liens de parenté en collatéralité (frère, cousin) et tous les liens de famille péri-incestueux échappent donc à cette nouvelle disposition du droit bien que le législateur ait prévu un large champ d'incrimination. Ce qui traduit, dans la pratique judiaicire, une conception minimaliste de l'inceste. Le législateur n'a-t-il pas échoué à vouloir rendre visible l'inceste dans un droit pénal qui peine à s'appliquer dans les tribunaux?

\subsubsection{Critère aggravant d'ascendant ou de personne ayant autorité : un usage disparate}

Le critère d'ascendant est toujours retenu par les professionnels lorsqu'un père ou un grandpère sont mis en cause. Dans ce cas, l'infraction est systématiquement aggravée. En revanche, le critère de personne ayant autorité est beaucoup plus problématique. L'autorité, n'étant pas définie par le droit, la doctrine jurisprudentielle fait référence. Elle a coutume de distinguer l'autorité de droit et l'autorité de fait. La première repose sur un lien légalement institué, tandis que la seconde, plus difficile à caractériser, résulte des circonstances qui ont mené à l'exercer sur l'enfant.

Or, dans notre corpus, presque la moitié des délits sexuels commis au sein de la famille ne sont pas aggravés, comme le montre le tableau ci-dessous :

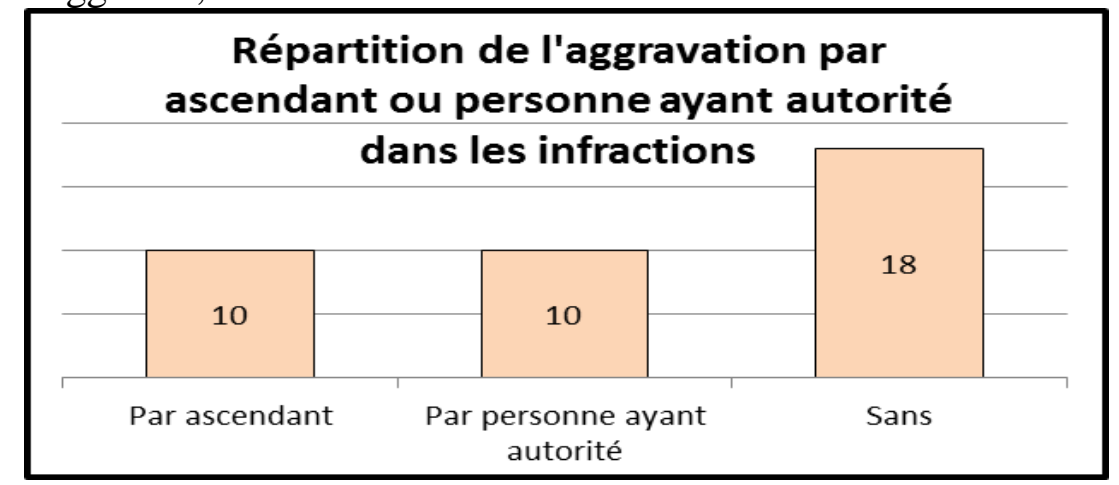

Prenons l'exemple de l'affaire G4. Aline dit avoir été victime à l'âge de 13 ans d'abus sexuel par son beau-père, 45 ans, vivant au sein du foyer recomposé depuis plus de dix ans. Le lien de famille est explicitement mentionné dans l'audition d'Aline «mon beau-père est apparu nu, il $m$ 'a saisi le poignet et a porté ma main à son sexe ${ }^{x v i i}$ », les procès-verbaux des enquêteurs "le mis en cause explique son geste par une mauvaise interprétation de sa belle-fille ${ }^{\text {xviii }}{ }$ et dans le rapport de fin d'enquête de police «le mis en cause se serait promené nu devant sa belle-fille âgée de 13 
ans ». Or, le beau-père n'a pas été reconnu comme personne ayant autorité, bien que les conditions d'autorité soient fondées par le vécu partagé. Les faits ont été qualifiés d'agression sexuelle sur mineur de 15 ans, sans tenir compte de ce lien factuel assimilé en anthropologie de la parenté au parent additionnel (Théry, 1991) ou aux co-résidents (Dussy, 2006).

Dans d'autres affaires, les juges n'ont pas reconnu la qualité d'autorité à de jeunes mis en cause (oncle et frère mineurs, cousin de 22 ans). Elle paraît difficile à reconnaître aux auteurs présumés mineurs ou très jeunes. S'agissant du concubin de la grand-mère, du père du concubin de la mère, du concubin de la sœur et des quasi-frères et sœur, les juges ne leur ont jamais reconnus l'autorité de fait. La répression de l'inceste s'avère donc incertaine aux confins de la parenté : on entre dans la complexité des familles recomposées, bien connue des anthropologues contemporains de la parenté (Cadolle, 2000 ; Martial, 1996, 1998 ; Poitevin, 2006).

La pratique judiciaire ne retient que les liens de parenté en ligne directe, ceux-là même qui paraissent susciter le plus d'effroi. L'apparition de nouveaux liens autour de l'enfant reste aux confins de l'incrimination pénale de l'inceste.

\subsection{Prouver le non-consentement du mineur victime d'inceste : prévalence de l'âge}

S'il peut paraitre incongru de se poser la question de la preuve du non-consentement du mineur victime d'inceste, il faut rappeler qu'en droit positif, le lien de famille entre auteur et victime n'a aucune incidence pour fonder l'agression sexuelle (Glandier, 2006). Dans nos affaires, cette qualification est surreprésentée, tandis que l'atteinte sexuelle sur mineurs, infraction la plus facile à caractériser ${ }^{\text {xix }}$, est quasi-absente, comme le montre le tableau ci-dessous :

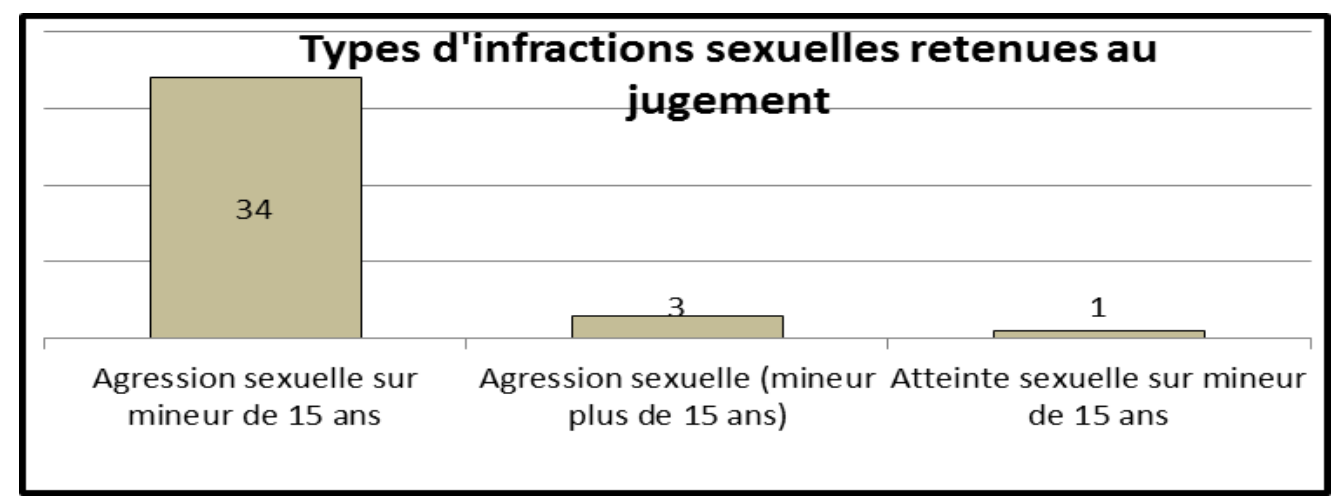

En droit, l'agression sexuelle implique, outre la nécessaire preuve matérielle des faits, qu'il y ait eu «violence, contrainte, menace ou surprise». Implicitement c'est la preuve du nonconsentement du mineur. Or, nos dossiers révèlent des particularités propres aux situations incestueuses bien connues des juristes (Vrignaud, 1994 ; Guéry, 1998 ; Porchy, 2003 ; Terryn, 2013) : la violence est peu commune, l'élément de surprise est rarement invoqué pour des faits incestueux qui se répètent et ne sont pas isolés, la menace est souvent postérieure aux actes pour garder le secret, la contrainte est quasiment toujours contestée par le mis en cause et celle prévue par le législateur (2010) n'a jamais été retenue. Alors sur quoi se fonde la preuve du nonconsentement du mineur?

Dans le corpus d'affaires, l'âge acquiert une place inédite étant à la fois critère aggravant et constitutif de la même infraction. Les trois affaires suivantes, impliquant des victimes présumées d'âges différents ( 7 ans, 13 ans et 15 ans), illustrent ce phénomène. 


\subsubsection{Le jeune âge de l'enfant : un seuil irréfragable de non-consentement}

La tendance de la doctrine jurisprudentielle est d'utiliser le critère d'âge pour constituer le non-consentement d'enfants très jeunes (moins de 6 ans), en raison de leur immaturité sexuelle ${ }^{x x}$. Cette présomption irréfragable de non-consentement statutaire lié à l'âge est présentée dans l'affaire suivante.

Affaire N28: "Tom, 7 ans, est victime d'attouchements sexuels par son quasi-frère, Bastien, 14 ans, enfant de son beau-père. Les faits sont avérés et reconnus à la fin de la procédure. Bastien est condamné pour agression sexuelle sur mineur de 15 ans à un mois d'emprisonnement avec sursis simple et à une mesure de liberté surveillée jusqu'à sa majorité ».

Les parents de Tom sont divorcés. Il vit avec sa mère et son nouveau compagnon, qui a deux enfants d'une précédente union, Bastien et Elodie. Les faits attestés au cours de l'enquête, se sont déroulés au domicile. Ils sont signalés au père de Tom dans une lettre anonyme. Le père se présente dès le lendemain au commissariat pour déposer plainte et Tom est auditionné : "Bastien il m'a sucé le zizi deux fois ... une fois dans la douche et une fois dans le lit ${ }^{x x i}{ }$. La première qualification retenue par les enquêteurs est celle de viol sur mineur de 15 ans, en marge du procès-verbal. Les faits sont rapidement reconnus par Bastien, lors de sa garde à vue, mais sur la base d'un jeu : «C'est depuis que Tom regarde la série Sex and the city avec mon père qu'il me pose des questions [...] il m'a demandé ... la question ... du coup sur la fellation. Il voulait savoir ce que c'était et ce que ça faisait. Que lui as-tu répondu? Au début je lui ai dit que ce n'était pas pour lui, et après il a commencé à me la poser de plus en plus souvent [...] c'est au bout d'un moment que j'ai fini par craquer et que je lui ai montré, ce que c'était une fellation pour qu'il me laisse tranquille ». Bastien assure que les faits se sont produit une seule fois, et reconnaît avoir fait une bêtise. Les faits sont alors requalifiés en agression sexuelle sur mineur de 15 ans, sans que cela change par la suite.

A l'issue de l'enquête de police, Bastien est mis en examen par le juge des enfants pour agression sexuelle sur mineur de 15 ans. Une mesure éducative pénale est prononcée et le juge requiert des investigations supplémentaires en vue d'obtenir des éléments de personnalité des protagonistes. Ces éléments n'ont pas pour but la recherche de preuve de culpabilité mais l'appréciation du mode de vie passé et actuel ${ }^{\text {xiii }}$. Ainsi, l'expertise psychiatrique réalisée sur Bastien s'avère préoccupante : "Il est incapable d'élaborer quoique ce soit sur la pulsion qui est ramenée au registre des gestes inappropriés [...] l'attitude de l'adolescent n'est pas très rassurante ». Tandis que l'expertise psychologique retient l'hypothèse d'une pulsion incontrôlée «que les faits s'inscrivent dans un contexte de promiscuité enfant/adolescent avec un accès peu contrôlé à des informations sexuelles non indiquées pour un enfant». Quant à l'expertise psychologique de Tom, il est relevé sa grande immaturité en matière sexuelle.

Au terme de deux ans de procédure, Bastien est renvoyé devant le tribunal pour enfant pour agression sexuelle sur mineur de 15 ans. Dans cette affaire, la preuve du non-consentement de Tom repose sur son très jeune âge et l'asymétrie d'âge avec Bastien, comme cela est indiqué dans le jugement : "L'hypothèse d'un acte sollicité consciemment par la jeune victime peut être formellement exclue eu égard aux déclarations précises et circonstanciées de l'enfant il ne peut raisonnablement être retenu un simple jeu sexuel entre les deux mineurs. En effet, il existe une dissymétrie dans la relation entre Bastien et Tom, le premier ayant le double de l'âge du second, ce dernier étant à 7 ans encore ignorant du caractère sexuel des actes subis alors que Bastien à 14 ans était suffisamment mature pour avoir accès à la notion d'interdit en matière sexuelle ${ }^{x x i i i}{ }_{{ }}$. Outre le phénomène de correctionnalisation du viol, il n'y a aucune référence explicite à la dimension incestueuse. La gravité ne tient pas au lien de famille mais à la question des âges. On va voir qu'elle s'avère plus délicate avec la catégorie d'âge des presque majeurs sexuellement : les 13-15 ans. 


\subsubsection{Autres âges de la minorité : incertitudes des seuils}

Dans les deux affaires suivantes, la preuve du non-consentement de victimes mineures âgées de 13 ans et 15 ans et demi est plus délicate. Le lien de famille est peu significatif.

Affaire G1 : "Angélique, 13 ans, révèle à sa sœur aînée, un an après les faits, avoir subi pendant plus de 9 mois des attouchements sexuels de la part de son beau-père André, 30 ans. Les faits sont avérés et reconnus par le mis en cause en fin de procédure ».

Les faits sont avérés puisqu'André les a reconnus très rapidement, mais il avance l'idée d'un consentement mutuel. Lors de son audition de garde à vue, André explique : "Cela a été mutuel, on était d'accord l'un et l'autre [...] Je regrette qu'Angélique ne dise pas toute la vérité, car elle m'est apparue consentante ${ }^{x x i v} \gg$. De même, lors de sa confrontation dans le cabinet du juge d'instruction : "Pourquoi vous accuse-t-elle de choses si graves alors qu'elle était consentante? Réponse d'André : Je pense qu'elle était consentante sur le moment, qu'ensuite elle a compris comme moi qu'elle avait fait une faute et comme moi qu'elle a eu des remords, c'est pour cela qu'elle $m$ 'accuse ${ }^{x x v}{ }^{\prime}$. Il évoque avec l'expert psychiatre une pulsion partagée : "je vivais chez une ex-petite amie, et un jour en s'amusant, avec sa fille, on a eu une pulsion partagée, une envie de part et d'autre. On s'est embrassé et il y a eu un dérapage». L'expert relève un problème de positionnement d'André : "le sujet reconnaît certains des faits reprochés, mais critique très partiellement son propre comportement. Ceci pourrait faire envisager l'application d'une injonction de soins ${ }^{x x v i}{ }_{»}$.

En revanche, Angélique conteste tout consentement de sa part. Lors de la confrontation elle indique "je n'ai jamais été consentante comme il l'a dit ». Par ailleurs, on trouve deux expertises psychologiques d'Angélique dans le dossier. La première relève l'absence de culpabilité et de souffrance psychique, ce qui dénote fortement avec la représentation classique que l'on peut se faire d'une victime d'inceste. La deuxième apporte des éléments d'explication à l'incongruité de son attitude : "Angélique avait accepté les actes sexuels dans un mouvement qui n'est pas très loin d'un renoncement dépressif [...] La renonciation à se défendre [...] qui n'empêche pas l'apparition d'un sentiment de honte qui lui-même vient à son tour "freiner » les velléités de dévoilement ${ }^{x x v i i}$ ».

L'enjeu de cette affaire tient à la délicate preuve du non-consentement d'Angélique, comme le relève le Procureur dans son réquisitoire : "Devant le juge d'instruction, André persistait à affirmer que la jeune fille était toujours consentante, André [...] mis en examen du chef d'agression sexuelle aggravée en raison de la contrainte que la jeune fille a nécessairement subie. Angélique avait entre 13 et 14 ans au moment des faits et que son agresseur était le concubin de sa mère ${ }^{x x v i i i}{ }_{\text {». }}$ André a été condamné par le tribunal correctionnel pour agression sexuelle sur mineur de 15 ans par personne ayant autorité à une peine sévère : 3 ans d'emprisonnement dont 18 mois avec sursis et mise à l'épreuve pendant 2 ans, ainsi qu'une inscription au FIJAIS ${ }^{\text {xxix }}$.

Dans cette affaire, la preuve de non-consentement repose sur la coexistence de critères d'âge de la victime (moins de 15 ans) et de qualité d'auteur (personne ayant autorité) au sein de la même infraction. C'est un concours de qualification, phénomène bien connu des juristes qui fait controverse dans la doctrine (Mayaud, 2004). Le rapport inégal adulte/mineur assimile la dimension incestueuse de cette affaire, dans la suivante, il est encore plus marqué.

Affaire N15: "Léa 15 ans et demi révèle immédiatement à sa tante des attouchements sexuels qu'elle vient de subir de la part de son grand-père paternel, Jacques. La tante accompagne sa nièce aux urgences pédiatriques, l'affaire est aussitôt signalée au Parquet ». 
L'enquête a établi la matérialité des faits : caresses sur la poitrine et le sexe, cunnilingus. Les enquêteurs questionnent Léa sur les circonstances des faits : "Comment tu as réagi ? Je ne comprenais pas, mais j'ai rien fait parce que je comprenais pas [...] Là il vient vers moi et il enlève le tee-shirt [...] Comment tu as réagi ? Je me suis crispée, je ne pouvais rien faire. Qu'est-ce que tu as ressenti quand il a fait ça? Ça me faisait mal, je savais pas ... je savais pas ... (commence à

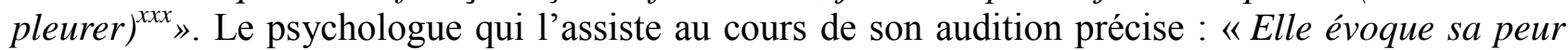
avec un effet de sidération. Elle ne savait pas quoi faire, laissant transparaître un sentiment de culpabilite $^{x x x i}{ }$. L'expertise psychologique de Léa mentionne une symptomatologie dépressive ancienne réactivée par les agressions sexuelles subies. Quant à l'expertise gynécologique elle atteste de l'absence d'hymen déchiré, ce qui infirme l'hypothèse d'un viol.

Bien que les faits soient rapidement reconnus par le grand-père paternel, âgé de 82 , ce dernier conteste toutefois la contrainte. Il évoque une séduction de la part de sa petite fille, posture critiquée par l'expert psychiatre : "On peut décrire chez lui des comportements pervers, qui l'ont amené à imposer à sa petite fille des attouchements sexuels qu'il reconnaît tout en les rationnalisant partiellement [...] On peut donc parler de pédophilie à caractère incestueux ${ }^{x x x i i}{ }$. La procédure est expéditive, deux jours d'enquête au terme desquels Jacques est renvoyé directement devant le tribunal correctionnel pour agression sexuelle par ascendant. La peine est sévère : 3 ans d'emprisonnement dont 24 mois de sursis avec interdiction de rencontrer la victime, d'exercer une activité même bénévole impliquant un contact habituel avec un mineur, suivi socio-judiciaire pendant 5 ans, inscription FIJAIS et privation de tous les droits civiques, civils et de famille pendant 5 ans. Statut de minorité et asymétrie d'âge prévalent ici, comme le relève la présidente de séance lors du procès : « «Je sais que tu en as envie " cela sort d'où ? Vous croyez qu'une fille de 15 ans a envie d'un vieux monsieur? Le prévenu : je ne sais pas. Ce n'est pas de ma faute, je ne sais pas ce qui m'a pris. Oui j'ai des regrets? J'avoue ce que j'ai fait ${ }^{\text {xxxiii }}{ }_{\gg .}$

La particularité dans cette affaire tient à sa qualification pénale : l'agression sexuelle. En l'absence de violence, contrainte, menace ou surprise, le plus simple pour les professionnels auraient été d'incriminer les faits en atteinte sexuelle sur mineur de 15 ans par ascendant ; seule incrimination spécifique de l'inceste dans le code pénal. Or, les juges ont opté pour faire du lien de famille et de la minorité, deux critères constitutifs et aggravants de la même infraction. L'inceste se trouve complètement dissout dans un rapport inégal adulte/mineur où prévaut un non-consentement statutaire lié à l'âge.

\section{Conclusion}

Cette première étude menée au sein de deux tribunaux pour enfants et correctionnels français nous a permis d'appréhender la complexité de la qualification pénale de l'inceste. Les dossiers judiciaires témoignent de l'établissement de seuils et de frontières incertains dès que l'on sort du seuil de parenté en ligne directe. Les liens de collatéralité ou issus des familles recomposées ne sont pas considérés comme des liens incestueux. En outre, il y a un vaste problème autour de la preuve du non-consentement du mineur victime d'inceste. Elle est extrêmement délicate à établir dans ces affaires sans violence, contrainte, menace ou surprise, où néanmoins, les seuils d'âge et les asymétries d'âge sont devenus de plus en plus importants.

Aussi, pour paradoxal que cela puisse paraître, nombre d'affaires échappent à la qualification pénale de l'inceste. On ne peut réduire l'explication au fait que le droit pénal peine à s'appliquer dans une structure complexe de la parenté telle que la nôtre, qui a connu des évolutions considérables ces quarante dernières années (Godelier, 2004 : 11). Sans doute, l'explication est à chercher ailleurs. En effet, si le lien de famille ne paraît pas déterminant dans les logiques d'incrimination, en revanche, il en est autrement du critère de l'âge, devenu essentiel, sinon 
primordial, comme preuve de non-consentement (Gouttenoire, 2008). Cette interprétation malléable qui se construit dans les palais de justice confirme l'émergence d'un non-consentement statutaire du mineur. Mais selon quels seuils d'âge de présomption?

Nos résultats tendent à montrer, que ce que l'on punit le plus sévèrement dans l'inceste est ce rapport inégalitaire majeur/mineur et plus particulièrement l'asymétrie d'âge et de sexe. Aussi, dans un système de parenté européen, où domine un principe de dominance sexuée, prime une logique de verticalisation des rapports, déjà à l'œuvre au sein des fratries (Héritier, 2010: 35). L'entrée de la catégorie de l'âge du mineur, dans une approche socio-anthropologique, est devenue un enjeu majeur de la qualification pénale de l'inceste. L'enquête réalisée permet d'ores et déjà de vérifier l'hypothèse fondamentale de recomposition en cours autour de la barrière sacralisée des âges. Dans ce nouvel ordre sexuel, le non-consentement statutaire lié à l'âge prévaut sur la dimension de l'inceste. La pratique judiciaire confirme le poids d'un nouvel ordre symbolique qui englobe l'interdit des places (symboliques) dans celui des âges (majeur/mineur).

\section{Bibliographie}

Ambroise-Rendu, Anne-Claude. 2012. «L'inceste doit-il être interdit par le droit ? Deux siècles d’incertitude (1810-2010) ». In Esprit. n॰5 (Mai).

Cadolle, Sylvie. 2000. Etre parent, être beau-parent. Paris, Odile Jacob.

Carbonnier, Jean. 1994. Sociologie juridique. 1 vol. Quadrige (Paris. 1981), Paris, PUF.

- 2002. Droit civil. La famille, l'enfant, le couple. Tome 2. PUF.

Courtois, Gérard. 2004. «Portalis et la prohibition de l'inceste ». In Droit et cultures. $\mathrm{n}^{\circ} 48$

Demartini. Anne-Emmanuelle. L'affaire Nozière. La parole sur l'inceste et sa réception sociale dans la France des années 1930. In Revue d'histoire moderne et contemporaine. Vol. 4. 2009. p 190 à 214

Dussy, Dorothée. 2006. «L'inceste versus l'interdit de l'inceste, lectures croisées ». Journal International de Victimologie 1.

- 2013a. Le Berceau des dominations. Anthropologie de l'inceste, livre 1. Marseille, La Discussion

- 2013b. (Dir.) L'inceste, bilan des savoirs. Marseille, La Discussion

Finkelhor, David et Jean, Giles-Sims. 1984. «Child abuse in Stepfamilies”. In Family relations. Vol. 33, No. 3, Remarriage and Stepparenting (Jul., 1984), pp. 407-413

Finkelhor, David. 1994. « Sexual abuse of children ». In The Future of children. Vol. 4. n`2.

Giuliani, Fabienne. 2014. Les liaisons interdites. Histoire de l'inceste au XIXe siècle. Paris, La Sorbonne.

Godelier, Maurice. 2004. Métamorphoses de la parenté. 1 vol. Champs. Essais, Flammarion.

Gouttenoire, Adeline ; Bonfils, Philippe. 2008. « Droits de l'enfant ». Recueil Dalloz.

Guéry, Christian. 1998. L’inceste : étude de droit comparé. D. 1998. Recueil Dalloz. 
Guinchard, Serge. Debard, Thierry. 2014. Lexique des termes juridiques 2014/2015. Edition Dalloz. $22^{\text {ème }}$ édition. Broché.

Héritier, Françoise. 1979. « Symbolique de l'inceste et de sa prohibition ». In Michel Izard et Pierre Smith. La fonction symbolique. Paris, Gallimard.

- $\quad$ 1996. Masculin, Féminin. La pensée de la différence. Paris, Odile Jacob.

- 2010. Le rapport frère/sœur, pierre de touche de la parenté. Edition Société d'Ethnologie

Knibiehler, Yvonne. 2001. Regards sur la sexualité familiale. Atteintes sexuelles sur enfants mineurs : propos de cliniciens et juristes engagés dans leur pratique auprès de ces enfants ». Vol. 1. Paris API

Le Goaziou, Véronique. 2011. Le viol, aspects sociologiques d'un crime: une étude de viols jugés en cour d'assises. Mission de recherche Droit et justice et Centre de recherches sociologiques sur le droit et les institutions pénales. 1 vol. Perspectives sur la justice. Paris, France.

Martial, Agnès. 1996. S'apparenter. Ethnologie des liens de famille recomposés. Paris. Maison des sciences de l'homme.

- 1998. «Partages et fraternité dans les familles recomposées ». In Agnès Fine (Dir). Adoptions. Ethnologie des parentés choisies. Paris, Maison des Sciences de l'homme.

Mayaud, Yves. 2004. «Les violences sexuelles, les qualifications relatives aux atteintes sexuelles ». Actualité Juridique Pénal, n¹ (janvier): 9-13.

Mayer, Daniel. 1981. Le nouvel éclairage donné au viol par la réforme du 23 décembre 1980. Chronique judiciaire.

- 1988. La pudeur du droit face à l'inceste. Chronique judiciaire.

Poitevin, Aude. 2006. Enfants de familles recomposées. Presses Universitaires de Rennes.

Porchy, Marie-Pierre. 2003. Les silences de la loi : un juge face à l'inceste. Hachette.

Porqueres i Gené, Enric. 2000. «Cognatisme et voies du sang: La créativité du mariage canonique ». L'Homme. n¹54, 155, Question de parenté. p 335-336.

- 2013. Porqueres i Gené, Enric, Wilgaux, Jérôme (et al.). «Les incertitudes de l'inceste : Autour de l'anthropologie symbolique de Françoise Héritier ». Incidence. n ${ }^{\circ}$. Coll. «Textes de références ». Editions du Félin

Poumarède, Jacques. 1987. "L'inceste et le droit bourgeois au XIX ${ }^{\mathrm{e}}$ siècle », Droit, histoire et sexualité. Textes réunis par Jacques Poumarède et Jean-Pierre Royer. Lille : Publications de l'espace juridique. pp213-228.

Rassat, Marie-Laure. 2008a. «Agressions sexuelles - Viol - Autres agressions sexuelles - Exhibition sexuelle - Harcèlement sexuel ». LexisNexis, ${ }^{\circ}{ }^{0}$ Version 1/2010.

- 2008b. «Mise en péril des mineurs - Atteintes sexuelles sans violence sur mineur Corruption de mineur - ». NexisLexis, n ${ }^{\circ} 1 / 2011$. NexisLexis.

Salas, Denis. 1996. «L’inceste, un crime généalogique ». Esprit n²27, pp. 122-136 
Terryn, Fabienne. 2013. «L'inceste en droit pénal français », in Dorothée Dussy (Dir.) L’inceste, bilan des savoirs. Marseille, La Discussion

Théry, Irène. 1991. «Trouver le mot juste : langage et parenté dans les recompositions familiales après divorce », in M. Segalen (dir.), Jeux de famille, Paris, Presses sur CNRS, pp. 5-21

- 1993. Le Démariage. Paris: Odile Jacob.

- 2002. «Les trois révolutions du consentement: Pour une approche socio anthropologique de la sexualité ». In XXXIIIe colloque français de criminologie. Actes. Dalloz.

- 2010. Des humains comme les autres. Bioéthique, anonymat et genre du don. Ecole des Hautes Etudes en Sciences sociales.

- 2016. Mariage et filiation pour tous. Une métamorphose inachevée. Seuil et République des idées.

Tournier, Victor. 2008. «Violence sexuelle. Approche de démographie pénale ». Débats. $\mathrm{n}^{\circ} 1$. Observatoire National de la Délinquance. (Janvier).

Vigarello, Georges. 1998. Histoire du viol : XVIe-XXe siècle. Editions du Seuil.

Vrignaud, Dominique. 1994. «Les comptes ordinaires de l'inceste », in Françoise Héritier (dir.), De l'inceste. Paris, Le seuil

\footnotetext{
${ }^{\mathrm{i}}$ Lexique des termes juridiques 2014/2015 Edition Dalloz. p 552

${ }^{\text {ii } L e ~ m e ̂ m e ~ c r i t e ̀ r e ~ e s t ~ c o n s t i t u t i f ~ d e ~ l ' a t t e i n t e ~ s e x u e l l e ~ s u r ~ m i n e u r ~ d e ~ p l u s ~ d e ~} 15$ ans, seule incrimination spécifique.

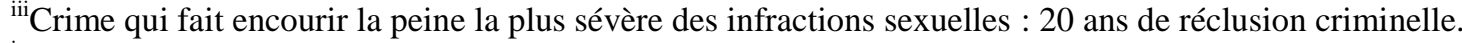

${ }^{\text {iv }}$ Cet article s'inscrit dans le cadre de la rédaction d'une thèse en sociologie judiciaire portant sur 80 affaires de violences sexuelles sur mineurs. Pour des raisons éthiques, les prénoms de tous les protagonistes ont été modifiés.

${ }^{\vee}$ Article 333 de l'ancien code pénal.

${ }^{\mathrm{vi}} 13$ ans en 1863 et 15 ans depuis 1945.

${ }^{\text {vii }}$ Seule exception du code à partir de 1863 : nouvel article 331 de l'ancien code pénal, alinéa 2, l'attentat à la pudeur sur mineur de plus de 13 ans par ascendant. Les deux critères sont constitutifs de l'incrimination.

${ }^{\text {viii }}$ Nouvel article 332 de l'ancien code pénal.

${ }^{\text {ix }}$ Article 222-23 du code pénal

${ }^{\mathrm{x}}$ Article 222-27 du code pénal

${ }^{\mathrm{xi}}$ Article $227-25$ du code pénal

${ }^{x i i}$ Articles 222-31-1 et 227-27-2 du code pénal «les viols, les agressions sexuelles et les atteintes sexuelles sont qualifiés d'incestueux lorsqu'ils sont commis au sein de la famille sur la personne d'un mineur par un ascendant, un frère, une sœur ou par toute autre personne, y compris s'il s'agit d'un concubin d'un membre de la famille, ayant sur la victime une autorité de droit ou de fait ».

${ }^{\text {xiii }}$ Suite à deux QPC, le 16 septembre 2011 et le 17 février 2012, le conseil constitutionnel a rejeté les articles en raison d'une trop grande imprécision du champ d'incrimination des auteurs. La loi n²016-297 du 14 mars 2016 , les a réintroduit sur une base plus conforme aux prohibitions matrimoniales du code civil.

${ }^{\mathrm{xiv}}$ Article 222-22 du code pénal «La contrainte peut être physique ou morale. Elle peut résulter de la différence d'âge existant entre une victime mineure et l'auteur des faits et de l'autorité de droit ou de fait que celui-ci exerce sur cette victime "

${ }^{\mathrm{xv}}$ Les enfants du conjoint d'un des parents, issus d'une précédente union

${ }^{\mathrm{xvi}}$ Dans le code civil, articles 161 à 164, le périmètre des interdictions matrimoniales est absolue à l'ascendant, au frère, à la sœur ; dispenses possibles à l'oncle ou la tante, le beau-père ou la belle-mère.

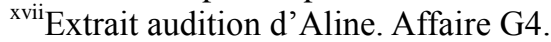

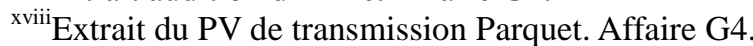

${ }^{x i x}$ Définit à l'inverse de l'agression sexuelle, c'est-à-dire sans violence, contrainte, menace ou surprise

${ }^{\mathrm{xx}}$ Cour de cassation du 7 décembre 2005.

${ }^{\mathrm{xxi}}$ Extrait première audition Tom. Affaire N28.
} 
${ }^{x x i i}$ Article D16 du code de procédure pénale.

${ }^{x x i i i}$ Extrait jugement. Affaire N28.

${ }^{x x i v}$ Extrait audition André. Affaire G1.

${ }^{\mathrm{xxv}}$ Extrait audience de confrontation. Affaire G1.

${ }^{\mathrm{x} x \mathrm{i}}$ Extrait expertise psychiatrique d'André. Affaire G1

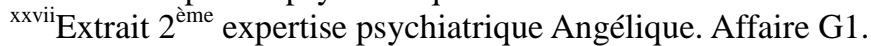

${ }^{x x v i i i}$ Extrait du réquisitoire. Affaire G1.

${ }^{x x i x}$ Fichier Judiciaire Automatisé des Auteurs d'Infractions Sexuelles

${ }^{\mathrm{xxx}}$ Extrait audition Léa. Affaire N15.

${ }^{\mathrm{xxxi}}$ Extrait compte rendu psychologique. Affaire N15.

${ }^{x x x i i}$ Extrait expertise psychiatrique Jacques. Affaire N15.

xxxiii Extrait notes d'audience. Affaire N15. 